\title{
Countable Toronto spaces
}

\author{
by \\ Gary Gruenhage (Auburn, AL) and J. Tach Moore (Toronto)
}

\begin{abstract}
A space $X$ is called an $\alpha$-Toronto space if $X$ is scattered of CantorBendixson rank $\alpha$ and is homeomorphic to each of its subspaces of the same rank. We answer a question of Steprāns by constructing a countable $\alpha$-Toronto space for each $\alpha \leq \omega$. We also construct consistent examples of countable $\alpha$-Toronto spaces for each $\alpha<\omega_{1}$.
\end{abstract}

1. Introduction. For a space $X$, let $X^{0}=X$, let $X^{\alpha+1}$ be the nonisolated points of $X^{\alpha}$, and for $\alpha$ a limit, let $X^{\alpha}=\bigcap_{\beta<\alpha} X^{\beta} . X$ is scattered if $X^{\alpha}=\emptyset$ for some $\alpha$. In this case, we call the least such $\alpha$ the CantorBendixson rank, $r(X)$, of $X$. The set $X^{\alpha} \backslash X^{\alpha+1}$ (i.e., the isolated points of $\left.X^{\alpha}\right)$ is called the $\alpha$ th level of $X$.

The so-called Toronto problem, posed by J. Steprāns [S], asks if there is an uncountable non-discrete Hausdorff space $X$ which is homeomorphic to each of its uncountable subspaces. Such a space, if it exists, is called a Toronto space. According to the folklore, a Toronto space must be scattered of Cantor-Bendixson rank $\omega_{1}$, and hereditarily separable (in particular, each level must be countable). The Toronto problem is still unsettled, though it is known that there are no Toronto spaces under $\mathrm{CH}$, and no regular Toronto spaces under PFA.

Taking a cue from the structure of a Toronto space, Steprāns calls a space $X$ an $\alpha$-Toronto space if $X$ is scattered of rank $\alpha$, and $X$ is homeomorphic to each of its subspaces of the same rank. For example, a convergent sequence is 2-Toronto. Steprāns asks if there is an $\omega$-Toronto space, and mentions that it is unknown if there is an $\alpha$-Toronto space for any $\alpha \geq 3$. The main result of this paper is that there is a countable $\alpha$-Toronto space for any $\alpha \leq \omega$, and there are consistent examples of countable $\alpha$-Toronto spaces for each $\omega<\alpha<\omega_{1}$.

2000 Mathematics Subject Classification: 54G12, 54G15, 54A35.

Research of the first author partially supported by NSF DMS-9704849. 
Given a filter $\mathcal{F}$ on $\omega$, let $\mathcal{F}^{+}$be the set of all $X \subset \omega$ such that $X \cap F \neq \emptyset$ for every $F \in \mathcal{F}$. $\mathcal{F}$ is said to be homogeneous if for any $X \in \mathcal{F}^{+}$, the restriction of the filter $\mathcal{F}$ to $X$ is isomorphic to $\mathcal{F}$. Let us also denote by $\mathcal{F} \times \omega$ the filter on $\omega \times \omega$ generated by sets of the form $F \times \omega, F \in \mathcal{F}$. We will show in Section 2 that if there is a homogeneous filter $\mathcal{F}$ on $\omega$ which is isomorphic to the filter $\mathcal{F} \times \omega$ on $\omega^{2}$, then there is an $\alpha$-Toronto space for every $\alpha \leq \omega$. If $\mathcal{F}$ has a certain additional property (see Theorem 2.11), then there is an $\alpha$-Toronto space for every $\alpha<\omega_{1}$.

In Section 3 we describe, in ZFC, a filter $\mathcal{F}$ which is homogeneous and isomorphic to $\mathcal{F} \times \omega$. Thus there are countable $\alpha$-Toronto spaces in ZFC for every $\alpha \leq \omega$. This filter does not have the additional property required to build countable $\alpha$-Toronto spaces for $\omega<\alpha<\omega_{1}$. In Section 4 , we show that it is consistent for there to be a filter having also the additional property, and hence consistent for there to be countable $\alpha$-Toronto spaces for every $\alpha<\omega_{1}$. We do not know if such filters (i.e., having the additional property) exist in ZFC, or if there can be countable $\alpha$-Toronto spaces in ZFC for $\omega<\alpha<\omega_{1}$.

Our construction also produces (consistent) $\alpha$-Toronto spaces for $\alpha=\omega_{1}$ and $\alpha=\omega_{1}+1$, albeit with uncountable levels. We do not know if there can be an $\omega_{1}$-Toronto space with countable levels.

In Section 5, we show that a very natural construction of spaces from a filter which is somewhat different from our construction in Section 2 cannot produce $\alpha$-Toronto spaces for $\alpha \geq 4$, though it does give other 3-Toronto spaces in ZFC.

2. Toronto spaces from the filter. Given a filter $\mathcal{F}$ on $\omega$, we will define corresponding scattered spaces $T_{\alpha}, \alpha$ an ordinal, of rank $\alpha . T_{\alpha}$ will be countable iff $\alpha<\omega_{1}$. Most other properties of the spaces $T_{\alpha}$ will depend on properties of the filter $\mathcal{F}$. We will show (Theorem 2.10) that if $\mathcal{F}$ is homogeneous and isomorphic to the filter $\mathcal{F} \times \omega$, then $T_{\alpha}$ is an $\alpha$-Toronto space for $\alpha \leq \omega$. If $\mathcal{F}$ has an additional property (see Theorem 2.11), then $T_{\alpha}$ is $\alpha$-Toronto for all $\alpha<\omega_{1}$.

If $\left(X_{n}\right)_{n \in \omega}$ is a sequence of (disjoint) spaces, and $\mathcal{F}$ is a filter on $\omega$, let $\left(X_{n}\right)_{n \in \omega} \cup_{\mathcal{F}}\{\infty\}$ denote the space whose set is $\{\infty\} \cup \bigcup_{n \in \omega} X_{n}$, such that each $X_{n}$ is a clopen subspace, and a neighborhood of $\infty$ has the form $\{\infty\} \cup$ $\bigcup_{n \in F} X_{n}$, where $F \in \mathcal{F}$. If $\mathcal{F}$ is a filter on a set $A$, then $\left(X_{a}\right)_{a \in A} \cup_{\mathcal{F}}\{\infty\}$ is defined similarly.

Let $T_{0}$ be the empty space and $T_{1}$ a single point space. Let $T_{\alpha+1}=$ $\left(\{n\} \times T_{\alpha}\right)_{n \in \omega} \cup_{\mathcal{F}}\{\infty\}$. If $\alpha$ is a limit ordinal and $T_{\beta}$ has been defined for all $\beta<\alpha$, let $T_{\alpha}=\bigoplus_{\beta<\alpha} T_{\beta}$. Clearly $T_{\alpha}$ is scattered of rank $\alpha$, and is countable iff $\alpha<\omega_{1}$. 
To verify other properties of the spaces $T_{\alpha}$, given properties of the filter $\mathcal{F}$, it will be helpful to establish some general facts about the " $\cup_{\mathcal{F}}$ " construction.

Lemma 2.1. If $W \subset\left(X_{n}\right)_{n \in \omega} \cup_{\mathcal{F}}\{\infty\}$, then

$$
\infty \in \overline{W \backslash\{\infty\}} \Leftrightarrow\left\{n: W \cap X_{n} \neq \emptyset\right\} \in \mathcal{F}^{+} .
$$

Pr o of. Clear.

LEMMA 2.2. If $h_{n}: X_{n} \rightarrow Y_{n}$ is a homeomorphism for each $n \in \omega$, then the map $H:\left(X_{n}\right)_{n \in \omega} \cup_{\mathcal{F}}\{\infty\} \rightarrow\left(Y_{n}\right)_{n \in \omega} \cup_{\mathcal{F}}\{\infty\}$ defined by $H(\infty)=\infty$ and $H(x)=h_{n}(x)$ for $x \in X_{n}$ is a homeomorphism.

Proof. That $H$ is a bijection and continuous at every point except (possibly) $\infty$ is clear. It follows from Lemma 2.1 that $\infty \in \bar{W} \Leftrightarrow \infty \in \overline{H(W)}$. Thus $H$ is also continuous at $\infty$. The proof that $H^{-1}$ is continuous is similar. Thus $H$ is a homeomorphism.

Lemma 2.3. For each $A \subset \omega,\left(X_{n}\right)_{n \in \omega} \cup_{\mathcal{F}}\{\infty\} \cong\left[\left(\left(X_{n}\right)_{n \in A} \cup_{\mathcal{F} \mid A}\right.\right.$ $\left.\{\infty\}) \oplus\left(\left(X_{n}\right)_{n \in \omega \backslash A} \cup_{\mathcal{F} \uparrow(\omega \backslash A)}\{\infty\}\right)\right] /\{\infty\}$.

Proof. That the obvious mapping is a homeomorphism follows easily from Lemma 2.1, upon noting that $B \in \mathcal{F}^{+}$iff either $B \cap A \in \mathcal{F}^{+}$or $B \cap(\omega \backslash A) \in \mathcal{F}^{+}$.

Another straightforward application of Lemma 2.1 shows:

Lemma 2.4. For spaces $X_{n}$ and $Y_{n}, n \in \omega$, we have

$$
\left(X_{n} \oplus Y_{n}\right)_{n \in \omega} \cup_{\mathcal{F}}\{\infty\} \cong\left[\left(\left(X_{n}\right)_{n \in \omega} \cup_{\mathcal{F}}\{\infty\}\right) \oplus\left(\left(Y_{n}\right)_{n \in \omega} \cup_{\mathcal{F}}\{\infty\}\right)\right] /\{\infty\} .
$$

Lemma 2.5. If $\mathcal{F}$ is homogeneous, then for any $A \in \mathcal{F}^{+}$, we have

$$
\left(X_{n}\right)_{n \in \omega} \cup_{\mathcal{F}}\{\infty\} \cong\left(Y_{n} \oplus Z_{n}\right)_{n \in \omega} \cup_{\mathcal{F}}\{\infty\}
$$

where $\left(Y_{n}\right)_{n \in \omega}$ is a reindexing of $\left(X_{n}\right)_{n \in A}$, and $Z_{n}=X_{n}$ for $n \in \omega \backslash A$ while $Z_{n}=\emptyset$ for $n \in A$.

Proof. By homogeneity, $\left(X_{n}\right)_{n \in A} \cup_{\mathcal{F} \mid A}\{\infty\}$ is homeomorphic to $\left(Y_{n}\right)_{n \in \omega} \cup_{\mathcal{F}}\{\infty\}$ for some reindexing $\left(Y_{n}\right)_{n \in \omega}$ of $\left(X_{n}\right)_{n \in A}$. Also, it is clear that $\left(X_{n}\right)_{n \in \omega \backslash A} \cup_{\mathcal{F} \backslash(\omega \backslash A)}\{\infty\}$ is homeomorphic to $\left(Z_{n}\right)_{n \in \omega} \cup_{\mathcal{F}}\{\infty\}$ where the $Z_{n}$ 's are as given in the statement of the lemma. The lemma now follows by applying Lemma 2.3, the above remarks, and Lemma 2.4 .

LEMma 2.6. If $\mathcal{F}$ is homogeneous and not the co-finite filter, then $T_{\alpha}$ is homeomorphic to every topological sum of $T_{\alpha}$ and countably many $T_{\beta}$ for $\beta<\alpha$. 
Proof. The result is obvious for $T_{0}$ and $T_{1}$. Suppose it holds for all $\beta<\alpha$. If $\alpha$ is a limit ordinal, then $T_{\alpha}$ is by definition the topological sum of the $T_{\beta}$ 's for $\beta<\alpha$, and the result follows easily from the induction hypothesis.

Suppose $\alpha$ is a successor, say $\alpha=\gamma+1$, and consider $T_{\alpha} \oplus \bigoplus_{n \in \omega} X_{n}$, where each $X_{n}$ is homeomorphic to some $T_{\beta_{n}}, \beta_{n}<\alpha$. Note that $\mathcal{F}$ homogeneous and not the co-finite filter implies that $T_{\alpha}$ is homeomorphic to the topological sum of itself and countably many copies $Y_{n}, n \in \omega$, of $T_{\gamma}$. Now $T_{\alpha} \oplus \bigoplus_{n \in \omega} X_{n} \cong\left[T_{\alpha} \oplus \bigoplus_{n \in \omega} Y_{n}\right] \oplus \bigoplus_{n \in \omega} X_{n} \cong T_{\alpha} \oplus \bigoplus_{n \in \omega}\left(X_{n} \oplus Y_{n}\right)$. By the induction hypothesis, each $X_{n} \oplus Y_{n}$ is homeomorphic to one or two copies of $T_{\gamma}$, and the result follows.

Lemma 2.7. For any filter $\mathcal{F}$ on $\omega$, and disjoint spaces $\left\{X_{n, m}: n, m \in \omega\right\}$,

$$
\left(X_{n, m}\right)_{(n, m) \in \omega^{2}} \cup_{\mathcal{F} \times \omega}\{\infty\} \cong\left(\bigoplus_{m \in \omega} X_{n, m}\right)_{n \in \omega} \cup_{\mathcal{F}}\{\infty\} .
$$

Proof. Note that for $A \subset \omega^{2}, A \in(\mathcal{F} \times \omega)^{+} \Leftrightarrow \pi_{1}(A) \in \mathcal{F}^{+}$, where $\pi_{1}$ is the projection onto the first coordinate. Now it is easy to use Lemma 2.1 to verify that the obvious mapping is a homeomorphism.

The next lemma shows that if in the definition of $T_{\alpha+1}$, each copy $\{n\} \times$ $T_{\alpha}$ of $T_{\alpha}$ is replaced by the sum of finitely many or countably infinitely many copies of $T_{\alpha}$, the result is homeomorphic to $T_{\alpha+1}$, provided $\mathcal{F}$ has the stated properties.

Lemma 2.8. If $\mathcal{F}$ is homogeneous and isomorphic to $\mathcal{F} \times \omega$, then

$$
\left(\{n\} \times T_{\alpha}\right)_{n \in \omega} \cup_{\mathcal{F}}\{\infty\} \cong\left(\bigoplus_{m<k_{n}}\{n, m\} \times T_{\alpha}\right)_{n \in \omega} \cup_{\mathcal{F}}\{\infty\},
$$

where $0<k_{n} \leq \omega$.

Proof. Let $A=\bigcup_{n}\{n\} \times k_{n}$. Then $A \in(\mathcal{F} \times \omega)^{+}$. By the assumptions on $\mathcal{F},(\mathcal{F} \times \omega)\lceil A$ is isomorphic to $\mathcal{F} \times \omega$ and $\mathcal{F}$. Use an isomorphism between $(\mathcal{F} \times \omega)\lceil A$ and $\mathcal{F}$ to constuct the natural bijection between the spaces. Then use $B \in\left((\mathcal{F} \times \omega)\lceil A)^{+} \Leftrightarrow \pi_{1}(B) \in \mathcal{F}^{+}\right.$to show via Lemma 2.1 that this bijection is a homeomorphism.

Lemma 2.9. Suppose $\mathcal{F}$ is homogeneous and isomorphic to $\mathcal{F} \times \omega$. If $A \in \mathcal{F}^{+}$, then

$$
\left(X_{n}\right)_{n \in \omega} \cup_{\mathcal{F}}\{\infty\} \cong\left(\bigoplus_{m \in \omega} Y_{n}^{m}\right)_{n \in \omega} \cup_{\mathcal{F}}\{\infty\},
$$

where $\left(Y_{n}^{m}\right)_{n, m \in \omega}$ is a reshuffling of the $X_{i}$ 's, and for each $n, \mid\left\{m: Y_{n}^{m}=X_{i}\right.$ for some $i \in A\} \mid=\omega$. 
Proof. We have

$$
\begin{aligned}
\left(X_{n}\right)_{n \in \omega} & \cup_{\mathcal{F}}\{\infty\} \\
& \cong\left[\left(\left(X_{n}\right)_{n \in A} \cup_{\mathcal{F} \backslash A}\{\infty\}\right) \oplus\left(\left(X_{n}\right)_{n \in \omega \backslash A} \cup_{\mathcal{F} \backslash(\omega \backslash A)}\{\infty\}\right)\right] /\{\infty\} \\
& \cong\left[\left(\left(\bigoplus_{m \in \omega} W_{n}^{m}\right)_{n \in \omega} \cup_{\mathcal{F}}\{\infty\}\right) \oplus\left(\left(Z_{n}\right)_{n \in \omega} \cup_{\mathcal{F}}\{\infty\}\right)\right] /\{\infty\},
\end{aligned}
$$

where the $W_{n}^{m}$ 's are a reindexing of the $X_{n}$ 's, $n \in A$, and the $Z_{n}$ are as in Lemma 2.5. The first homeomorphism exists by Lemma 2.3, and the second follows from $\mathcal{F}$ being homogeneous and isomorphic to $\mathcal{F} \times \omega$, and Lemma 2.7. Now by Lemma 2.4, the latter quotient space is homeomorphic to $\left(Z_{n} \oplus\left(\bigoplus_{m \in \omega} W_{n}^{m}\right)\right)_{n \in \omega} \cup_{\mathcal{F}}\{\infty\}$. Finally, for each $n$ let $Y_{n}^{m}, m \in \omega$, be a reindexing of $\left\{Z_{n}\right\} \cup\left\{W_{n}^{m}: m \in \omega\right\}$.

THEOREM 2.10. If $\mathcal{F}$ is homogeneous and isomorphic to $\mathcal{F} \times \omega$, then $T_{n}$ is a countable $n$-Toronto space for every $n \leq \omega$, and any subspace of $T_{n}$ of rank $j<n$ is homeomorphic to a topological sum of countably many copies of $T_{j}$.

Proof. Clearly the theorem holds for $n \leq 1$. Also, if it holds for all $n<\omega$, it is easy to check that it holds for $n=\omega$, since $T_{\omega}$ is just the topological sum of the $T_{n}$ 's, $n<\omega$.

We will complete the proof by showing that the theorem holds for $n=$ $k+1$, given it holds for $n \leq k$, where $k<\omega$. To this end, let $X$ be a subspace of $T_{k+1}$. If $\infty$ is either not in $X$ or is an isolated point of $X$, it is easy to use the induction hypothesis to verify the conclusion of the theorem. So, suppose $\infty$ is a limit point of $X$. Let $X_{n}=X \cap\left(\{n\} \times T_{k}\right)$. Note that $X \cong\left(X_{n}\right)_{n \in \omega} \cup_{\mathcal{F}}\{\infty\}$. Let $j_{0} \leq k$ be maximal such that $\left\{n: r\left(X_{n}\right)=\right.$ $\left.j_{0}\right\} \in \mathcal{F}^{+}$. Then there is $F_{0} \in \mathcal{F}$ such that $j_{0}=\max \left\{r\left(X_{n}\right): n \in F_{0}\right\}$, and $A_{0}=\left\{n \in F_{0}: r\left(X_{n}\right)=j_{0}\right\} \in \mathcal{F}^{+}$. Let $N_{0}$ be the clopen neighborhood $\{\infty\} \cup \bigcup_{n \in F_{0}} X_{n}$ of $\infty$ in $X$; note $N_{0} \cong\left(X_{n}\right)_{n \in F_{0}} \cup_{\mathcal{F}}\{\infty\}$. By homogeneity and Lemma 2.5, $N_{0}$ is homeomorphic to $\left(Y_{n} \oplus Z_{n}\right)_{n \in \omega} \cup_{\mathcal{F}}\{\infty\}$ where $\left(Y_{n}\right)_{n \in \omega}$ is a reindexing of $\left(X_{n}\right)_{n \in A_{0}}$, and each $Z_{n}$ is either $\emptyset$ or $X_{m}$ for some $m \in$ $F_{0} \backslash A$. By the induction hypothesis, since $r\left(Z_{n}\right) \leq r\left(Y_{n}\right)=j_{0}$, each $Y_{n} \oplus Z_{n}$ is a topological sum of countably many copies of $T_{j_{0}}$. So by Lemma $2.8, N_{0}$ is homeomorphic to $T_{j_{0}+1}$. Let $N_{1}=X \backslash N_{0}$. By the induction hypothesis, $N_{1}$ is homeomorphic to a topological sum of countably many copies of $T_{j_{1}}$ for some $j_{1} \leq k$. Of course $X \cong N_{0} \oplus N_{1}$. If $j_{0}=k$, which happens iff $r(X)=k+1$, then $N_{0}$ and (by Lemma 2.6) $X$ are homeomorphic to $T_{k+1}$. If $j_{0}<k$, then by Lemma 2.6, $X$ is homeomorphic to a topological sum of countably many copies of $T_{\max \left\{j_{0}+1, j_{1}\right\}}$. That completes the proof.

TheOREM 2.11. Suppose $\mathcal{F}$ is homogeneous and isomorphic to $\mathcal{F} \times \omega$, and satisfies 
(*) Whenever $f: \omega \rightarrow \omega$ is unbounded on every $F \in \mathcal{F}$, there is some $A \in \mathcal{F}^{+}$such that $f\lceil A$ is finite-to-one.

Then $T_{\alpha}$ is a countable $\alpha$-Toronto space for every $\alpha<\omega_{1}$, and any subspace of $T_{\alpha}$ of rank $\beta<\alpha$ is homeomorphic to a topological sum of countably many copies of $T_{\beta}$.

Proof. Theorem 2.10 shows this theorem holds for $\alpha \leq \omega$. Suppose it holds for all $\beta<\alpha$, where $\alpha<\omega_{1}$, and consider $X \subset T_{\alpha}$.

If $\alpha$ is a limit, then $T_{\alpha}=\bigoplus_{\beta<\alpha} T_{\beta}$. By the induction hypothesis, each subspace $X \cap T_{\beta}$ of $X$ is homeomorphic to the topological sum of copies of $T_{\beta^{\prime}}$ for some $\beta^{\prime}<\beta$. Now one can use Lemma 2.6 to split up or group these $T_{\beta}$ 's in the appropriate way to show that $X \cong T_{r(X)}$ if $r(X)$ is a limit ordinal, and is homeomorphic to the sum of countably many copies of $T_{r(X)}$ otherwise.

It remains to verify the theorem in case $\alpha$ is a successor, say $\alpha=\gamma+1$. As in the proof of Theorem 2.10, we may suppose that $\infty$ is a limit point of $X$, in which case $X \cong\left(X_{n}\right)_{n \in \omega} \cup_{\mathcal{F}}\{\infty\}$, where $X_{n}=X \cap\left(\{n\} \times T_{\gamma}\right)$.

Let $\delta_{n}=r\left(X_{n}\right)$, and let $\delta$ be minimal such that, for some $F_{0} \in \mathcal{F}$,

$$
\sup \left\{\delta_{n}: n \in F_{0}\right\}=\delta .
$$

Let $A=\left\{n \in F_{0}: \delta_{n}=\delta\right\}$. If $A \in \mathcal{F}^{+}$, let $A_{0}=A$. If $A \notin \mathcal{F}^{+}$, then we may assume $A=\emptyset$, i.e., $\delta_{n}<\delta$ for each $n \in F_{0}$. Note that therefore $\delta$ is a limit ordinal. Let $\beta_{n}, n \in \omega$, be increasing with supremum $\delta$. Define $f: \omega \rightarrow \omega$ such that $f(n)=m$ iff $\beta_{m} \leq \delta_{n}<\beta_{m+1}$. By minimality of $\delta, f$ is unbounded on every $F \in \mathcal{F}$. By $(*)$, there is $A_{0} \in \mathcal{F}^{+}, A_{0} \subset F_{0}$, such that $f \nmid A_{0}$ is finite-to-one.

Thus, whether $A \in \mathcal{F}^{+}$or not, we have an $A_{0} \in \mathcal{F}^{+}, A_{0} \subset F_{0}$, such that $\sup \left\{\delta_{n}: n \in B\right\}=\delta$ for every infinite $B \subset A_{0}$. Let $N_{0}$ be the clopen neighborhood $\{\infty\} \cup \bigcup_{n \in F_{0}} X_{n}$ of $\infty$ in $X$. By homogeneity and Lemma 2.9, $N_{0} \cong\left(Z_{n}\right)_{n \in \omega} \cup_{\mathcal{F}}\{\infty\}$, where each $Z_{n}$ is a topological sum of $X_{m}$ 's, $m \in F_{0}$, with $m \in A_{0}$ infinitely often. Thus $r\left(Z_{n}\right)=\delta$ for all $n$. By the induction hypothesis, $Z_{n}$ is homeomorphic to a topological sum of countably many copies of $T_{\delta}$, hence by Lemma $2.8, N_{0} \cong T_{\delta+1}$. The proof is now completed as in Theorem 2.10.

We now show that the condition $(*)$ is necessary for $T_{\omega+1}$ to be $(\omega+1)$ Toronto.

TheOREM 2.12. Suppose $\mathcal{F}$ is a filter on $\omega$ which fails to satisfy condition (*) of Theorem 2.11. Then $T_{\omega+1}$ is not $(\omega+1)$-Toronto.

Proof. Recall $T_{\omega+1}=\left(\{n\} \times T_{\omega}\right)_{n \in \omega} \cup_{\mathcal{F}}\{\infty\}$. Suppose $T_{\omega+1}$ is $(\omega+1)$ Toronto, and let $f: \omega \rightarrow \omega$ be such that $|f(F)|=\omega$ for every $F \in \mathcal{F}$. We will show that $f\left\lceil A\right.$ is finite-to-one for some $A \in \mathcal{F}^{+}$. To this end, let 
$X=\left(\{n\} \times T_{f(n)}\right)_{n \in \omega} \cup_{\mathcal{F}}\{\infty\}$. Then $X$ is homeomorphic to a subspace of $T_{\omega+1}$, and it follows from the fact that $|f(F)|=\omega$ for every $F \in \mathcal{F}$ that $r(X)=\omega+1$.

So there must exist a homeomorphism $h: T_{\omega+1} \rightarrow X$. For $k \in \omega$ let

$$
B_{k}=\left\{n \in \omega: h\left(\{k\} \times T_{\omega}\right) \cap\left(\{n\} \times T_{f(n)}\right) \neq \emptyset\right\} .
$$

Since $r\left(T_{\omega}\right)=\omega$, we must have $\left|f\left(B_{k}\right)\right|=\omega$. Thus we can choose $n_{k} \in B_{k}$ such that $f\left(n_{0}\right)<f\left(n_{1}\right)<\ldots$ Now pick $x_{k} \in h\left(\{k\} \times T_{\omega}\right) \cap\left(\left\{n_{k}\right\} \times T_{f\left(n_{k}\right)}\right)$. Note that $\infty \in \operatorname{cl}\left(h^{-1}\left(\left\{x_{k}: k \in \omega\right\}\right)\right)$. It follows that $A=\left\{n_{k}: k \in \omega\right\}$ is in $\mathcal{F}^{+}$. Since $f$ is one-to-one on $A$, that completes the proof.

THEOREM 2.13. If $\mathcal{F}$ satisfies the conditions of Theorem 2.11, then $T_{\omega_{1}}$ and $T_{\omega_{1}+1}$ are $\omega_{1}$-Toronto and $\left(\omega_{1}+1\right)$-Toronto, respectively (albeit with uncountable levels $)$. However, $T_{\omega_{1}+2}$ is not $\left(\omega_{1}+2\right)$-Toronto.

Proof. That $T_{\omega_{1}}$ is $\omega_{1}$-Toronto follows just like the limit case of the proof of Theorem 2.11. Now consider a subspace $X$ of $T_{\omega_{1}+1}$ of rank $\omega_{1}+1$, and let $X_{n}=X \cap\left(\{n\} \times T_{\omega_{1}}\right)$. Then for $\mathcal{F}^{+}$-many $n$ 's, $r\left(X_{n}\right)=\omega_{1}$ and for such $n, X_{n} \cong T_{\omega_{1}}$. Now use Lemmas 2.5 and 2.8 as in Theorem 2.10 to complete the proof that $X \cong T_{\omega_{1}+1}$. Thus $T_{\omega_{1}+1}$ is $\left(\omega_{1}+1\right)$-Toronto.

Let $Y$ be the subspace of $T_{\omega_{1}+1}$ consisting of only its isolated points and the point $\infty$. Note that every neighborhood of $\infty$ in $Y$ is uncountable. It follows that $T_{\omega_{1}+2}$ has a subspace $Z$ of rank $\omega_{1}+2$ with a point at level 1 every neighborhood of which is uncountable. But every level 1 point of $T_{\omega_{1}+2}$ has a countable neighborhood. Thus $Z ¥ T_{\omega_{1}+2}$ and so $T_{\omega_{1}+2}$ is not $\left(\omega_{1}+2\right)$-Toronto.

3. The ZFC filter. In this section we will define a homogeneous filter $\mathcal{F}$ on $\omega$ such that $\mathcal{F}$ is isomorphic to $\mathcal{F} \times \omega$. It will be more convenient for our purposes to actually define the filter on the countable ordinal $\omega^{\omega}=\sum_{n} \omega^{n}$. The filter $\mathcal{F}$ is the collection of all $A \subseteq \omega^{\omega}$ such that the order type of $\omega^{\omega} \backslash A$ is less than $\omega^{\omega}$. Note that the $\mathcal{F}$-positive subsets of $\omega^{\omega}$ are simply those which have order type $\omega^{\omega}$.

TheOREM 3.1. $\mathcal{F}$ is homogeneous and isomorphic to $\mathcal{F} \times \omega$.

Proof. Notice that if $X \subseteq \omega^{\omega}$ is in $\mathcal{F}^{+}$and $\Phi: X \rightarrow \omega^{\omega}$ is an order isomorphism then $\Phi$ is also an isomorphism between $\mathcal{F}\lceil X$ and $\mathcal{F}$. Thus $\mathcal{F}$ is homogeneous.

To see that $\mathcal{F}$ is isomorphic to $\mathcal{F} \times \omega$, recall that the ordinal $\omega \cdot \omega^{\omega}$ is the order type of the set $\omega \times \omega^{\omega}$ equipped with the lexicographical order. It is easy to see that

$$
\omega \cdot \omega^{\omega}=\sum_{n=1}^{\infty} \omega \cdot \omega^{n}=\omega^{\omega} .
$$


Let $\Phi$ be an order isomorphism between $\omega^{\omega} \times \omega$ with the reverse lexicographical order and $\omega^{\omega}$. Define $\mathcal{F}^{*}$ to be the preimage of $\mathcal{F}$ under $\Phi$. Now it suffices to show that $\mathcal{F}^{*}$ is equal to $\mathcal{F} \times \omega$. It should be clear that $\mathcal{F}^{*}$ contains $\mathcal{F} \times \omega$. If $A$ is in $\mathcal{F}^{*}$ then define $A^{0}$ to be the union of all $E \subseteq A$ such that $E=\pi^{-1}(\pi(E))$ where $\pi: \omega^{\omega} \times \omega \rightarrow \omega^{\omega}$ is the projection onto the first coordinate. Notice that $A^{0}$ is also in $\mathcal{F}$ since if the complement of $A$ has order type $\alpha<\omega^{\omega}$ then the complement of $A^{0}$ has order type at most $\omega \cdot \alpha<\omega^{\omega}$. Since $\pi\left(A^{0}\right)$ must be in $\mathcal{F}$, and since $\pi^{-1}\left(\pi\left(A^{0}\right)\right)=A^{0}, A \supseteq A^{0}$ is in $\mathcal{F} \times \omega$ and we are finished.

Corollary 3.2. There are, in $Z F C, n$-Toronto spaces for every $n \leq \omega$.

Pr o of. Immediate from Theorems 3.1 and 2.10.

Unfortunately, this filter does not produce $\alpha$-Toronto spaces for $\alpha>\omega$ by Theorem 2.12 and:

Proposition 3.3. Let $\mathcal{F}$ be the filter of Theorem 3.1. Then $\mathcal{F}$ does not satisfy condition $(*)$ of Theorem 2.11 .

Proof. Define $f: \omega^{\omega} \rightarrow \omega$ by $f(\alpha)=k$ iff $\alpha \in\left[\omega^{k}, \omega^{k}+\omega\right)$. If the restriction of $f$ to a set $A \subset \omega^{\omega}$ is finite-to-one, clearly $A$ has order type $\omega$ and hence is not in $\mathcal{F}^{+}$.

In the next section, we will show that at least there are consistent examples of filters satisfying all the conditions of Theorem 2.11.

4. The consistent filter. The purpose of this section is to prove the following.

THEOREM 4.1. If ZFC is consistent, then it is consistent with ZFC that there is a filter $\mathcal{F}$ on $\omega$ satisfying:

(i) $\mathcal{F}$ is homogeneous;

(ii) $\mathcal{F}$ is isomorphic to the filter $\mathcal{F} \times \omega$ on $\omega^{2}$;

(iii) Whenever $f: \omega \rightarrow \omega$ is unbounded on every $F \in \mathcal{F}$, then there is some $A \in \mathcal{F}^{+}$such that $f\lceil A$ is finite-to-one.

The starting point for our construction is the following observation. Suppose $\mathcal{F}_{e}=\left\{F_{e \alpha}: \alpha<\omega_{1}\right\}, e=0,1$, are subbases for filters on $\omega$ such that, whenever $H$ and $K$ are disjoint finite subsets of $\omega_{1}$ and $\bigcap_{\alpha \in H} F_{e \alpha} \backslash \bigcup_{\beta \in K} F_{e \beta}$ is non-empty, then it is infinite, and so is the corresponding set using $\mathcal{F}_{1-e}$. Then there is a natural $\sigma$-centered poset $P$ forcing $\mathcal{F}_{0}$ and $\mathcal{F}_{1}$ to be isomorphic: namely, $P$ consists of all pairs $p=\left(\tau^{p}, H^{p}\right)$, where $\tau^{p}$ is a finite one-to-one function from $\omega$ to $\omega$, and $H^{p} \in\left[\omega_{1}\right]^{<\omega}$. Declare $q \leq p$ if $\tau^{q} \supset \tau^{p}$, $H^{q} \supset H^{p}$, and for each $\beta \in H^{p}$, we have $n \in F_{0 \beta} \Leftrightarrow \tau^{q}(n) \in F_{1 \beta}$ whenever $n \in \operatorname{dom}\left(\tau^{q} \backslash \tau^{p}\right)$. This forcing adds a function $t: \omega \rightarrow \omega$ such that 
$t\left(F_{0 \beta}\right)={ }^{*} F_{1 \beta}$ for every $\beta<\omega_{1}$, and it is easy to see that such a function $t$ is an isomorphism.

Call a pair of filters having subbases as above a good pair. Any pair of filters generated by $\omega_{1}$-sized independent families is a good pair. Our naive idea to start with a filter $\mathcal{F}$ on $\omega$ generated by an independent family $\mathcal{A}=\left\{A_{\alpha}: \alpha<\omega_{1}\right\}$, consisting of $\omega_{1}$-many Cohen reals, then force it to be isomorphic to the filter $\mathcal{F} \times \omega$ on $\omega^{2}$, which is generated by the independent family $\{A \times \omega: A \in \mathcal{A}\}$, and finally iterate the type of poset described in the previous paragraph $\omega_{1}$ times (we start with a model of $\mathrm{CH}$ ) to force $\mathcal{F}$ to be homogeneous.

Note that for any infinite subset $X$ of $\omega$ in the ground model, the restriction of $\mathcal{A}$ to $X$ is still an independent family. The problem one runs into, however, is that this is not true for many subsets $X$ added by the forcings. For example, if some infinite set $X$ is added which is almost contained in every member of $\mathcal{A}$, then the restriction of $\mathcal{F}$ to $X$ is the cofinite filter, and then there is no hope of making $\mathcal{F}$ homogeneous. So we must in particular show sets like this are not added. In fact, we show that for any subset $X$ of $\omega$ added at some stage of the iteration, if $X \in \mathcal{F}^{+}$, then there is some $\delta<\omega_{1}$ such that the restriction of $\left\{A_{\alpha}: \alpha \geq \delta\right\}$ to $X$ is an independent family. By Lemma 4.2 below, this turns out to be enough for $\mathcal{F}$ and its restriction to $X$ to be a good pair of filters (witnessed by the subbase $\left\{\bigcap_{\phi(\alpha)<n} A_{\alpha}\right\}_{n<\omega} \cup\left\{A_{\alpha}: \alpha \geq \delta\right\}$ for $\mathcal{F}$ and its restriction to $X$ for $\mathcal{F}\lceil X)$, and so they can be forced to be isomorphic.

Forcing notation follows Kunen $[\mathrm{Ku}]$; in particular, $\operatorname{Fn}(X, Y)$ denotes the set of all functions from a finite subset of $X$ into $Y$. For $A \subset \omega$, we let $A^{1}=A$ and $A^{0}=\omega \backslash A$.

Lemma 4.2. Let $\mathcal{A}=\left\{A_{\alpha}: \alpha<\omega_{1}\right\}$ be an independent family of subsets of $\omega$, and let $\mathcal{F}$ be the filter generated by $\mathcal{A}$. Given $\varrho \in \operatorname{Fn}\left(\omega_{1}, 2\right)$, let $L_{\varrho}=$ $\bigcap_{\alpha \in \operatorname{dom}(\varrho)} A_{\alpha}^{\varrho(\alpha)}$. Suppose the following holds:

$$
\forall X \subset \omega\left[X \in \mathcal{F}^{+} \Rightarrow \exists \gamma<\omega_{1}\left(X \cap L_{\varrho} \neq \emptyset \text { for all } \varrho \in \operatorname{Fn}\left(\omega_{1} \backslash \gamma, 2\right)\right)\right] .
$$

(To express the property in words, one might say it means that the restriction of $\mathcal{A}$ to any member of $\mathcal{F}^{+}$is "eventually independent".) Then for every $X \in \mathcal{F}^{+}$, there exists $\delta<\omega_{1}$ and a finite-to-one $\phi: \delta \rightarrow \omega$ such that

$$
\left[\bigcap_{\phi(\alpha)<n} A_{\alpha} \backslash \bigcap_{\phi(\alpha) \leq n} A_{\alpha}\right] \cap L_{\varrho} \cap X \neq \emptyset
$$

for all $n<\omega$ and $\varrho \in \operatorname{Fn}\left(\omega_{1} \backslash \delta, 2\right)$.

Proof. Let $X \in \mathcal{F}^{+}$. Let $M$ be a countable elementary submodel containing $\mathcal{A}, X$, and a function $Y \mapsto \gamma(Y) \in \omega_{1}$ witnessing the hypothesized property. Let $\delta=M \cap \omega_{1}$. Construct a finite-to-one fuction $\phi: \delta \rightarrow \omega$ such 
that, for each $n, \phi^{-1}(n) \not \subset \gamma\left(X \cap \bigcap_{\phi(\alpha)<n} A_{\alpha}\right)$ as follows. Let $\delta=\left\{\delta_{0}, \delta_{1}, \ldots\right\}$. Note that $A_{\alpha} \in M$ for each $\alpha<\delta$, and $\gamma(Y)<\delta$ for each $Y \in M \cap \mathcal{F}^{+}$. Hence we can inductively choose a finite subset $\phi^{-1}(n)$ of $\delta$ containing:

(1) $\delta_{i}$, where $i$ is least such that $\delta_{i} \notin \bigcup_{j<n} \phi^{-1}(j)$;

(2) $\delta_{k}>\gamma\left(X \cap \bigcap_{\phi(\alpha)<n} A_{\alpha}\right)$.

We claim that this $\delta$ and $\phi$ have the desired properties. To see this, fix $n \in \omega$. Let $Z_{n}=\left[\bigcap_{\phi(\alpha)<n} A_{\alpha} \backslash \bigcap_{\phi(\alpha) \leq n} A_{\alpha}\right] \cap X$, and let $\varrho \in \mathrm{Fn}\left(\omega_{1} \backslash \delta, 2\right)$. We need to show $Z_{n} \cap L_{\varrho} \neq \emptyset$. Choose $\alpha^{\prime} \in \phi^{-1}(n) \backslash \gamma\left(X \cap \bigcap_{\phi(\alpha)<n} A_{\alpha}\right)$. Let $\varrho^{\prime}=\varrho \frown\left\langle\alpha^{\prime}, 0\right\rangle$. Since $\varrho^{\prime} \in \operatorname{Fn}\left(\omega_{1} \backslash \gamma\left(X \cap \bigcap_{\phi(\alpha)<n} A_{\alpha}\right), 2\right)$, we have

$$
\begin{aligned}
\emptyset \neq\left[X \cap \bigcap_{\phi(\alpha)<n} A_{\alpha}\right] \cap L_{\varrho^{\prime}} & =\left[\left(X \cap \bigcap_{\phi(\alpha)<n} A_{\alpha}\right) \backslash A_{\alpha^{\prime}}\right] \cap L_{\varrho} \\
& \subset\left[\left(X \cap \bigcap_{\phi(\alpha)<n} A_{\alpha}\right) \backslash \bigcap_{\phi(\alpha) \leq n} A_{\alpha}\right] \cap L_{\varrho} \\
& =Z_{n} \cap L_{\varrho} .
\end{aligned}
$$

Now we describe the posets that will be used in the iteration. Let $\mathcal{F}$ be the filter generated by an independent family $\mathcal{A}=\left\{A_{\alpha}: \alpha<\omega_{1}\right\}$, let $X \in \mathcal{F}^{+}$, let $\delta<\omega_{1}$, and let $\phi: \delta \rightarrow \omega$ be a finite-to-one function satisfying the conclusion of Lemma 4.2. Let $Q(\mathcal{A}, X, \delta, \phi)$ be the poset consisting of all $p=\left\langle\tau^{p}, F^{p}, n^{p}\right\rangle$ such that:

(a) $\tau^{p}$ is a finite one-to-one function from $\omega$ to $X$;

(b) $F^{p} \in\left[\omega_{1} \backslash \delta\right]^{<\omega}$;

(c) $n^{p} \in \omega$.

Define $q \leq p$ iff $\tau^{q} \supset \tau^{p}, F^{q} \supset F^{p}, n^{q} \geq n^{p}$, and

(i) $\forall n \in \operatorname{dom}\left(\tau^{q} \backslash \tau^{p}\right) \forall \beta \in F^{p}\left[n \in A_{\beta} \Leftrightarrow \tau^{q}(n) \in A_{\beta}\right]$;

(ii) $\forall n \in \operatorname{dom}\left(\tau^{q} \backslash \tau^{p}\right) \forall k \leq n^{p}\left[n \in \bigcap_{\phi(\alpha)<k} A_{\alpha} \Leftrightarrow \tau^{q}(n) \in \bigcap_{\phi(\alpha)<k} A_{\alpha}\right]$.

Lemma 4.3. The poset $Q=Q(\mathcal{A}, X, \delta, \phi)$ is $\sigma$-centered, and if $G$ is a $Q$-generic filter, then $t=\bigcup\left\{\tau^{p}: p \in G\right\}$ is a bijection from $\omega$ to $X$ such that $t\left(A_{\beta}\right)={ }^{*} A_{\beta} \cap X$ for all $\beta \geq \delta$, and $t\left(\bigcap_{\phi(\alpha)<n} A_{\alpha}\right)={ }^{*} X \cap \bigcap_{\phi(\alpha)<n} A_{\alpha}$ for all $n<\omega$. In particular, $t$ witnesses that in $V[G], \mathcal{F}$ is isomorphic to its restriction to $X$.

Proof. Clearly any two conditions $p$ and $q$ for which $\tau^{p}=\tau^{q}$ are compatible, and so the poset is $\sigma$-centered.

Let $G$ be a $Q$-generic filter. First we show that, for each $k \in \omega$, the subset of $Q$ consisting of all $p$ with $k \in \operatorname{dom}\left(\tau^{p}\right)$ is dense. To this end, suppose $k \notin \operatorname{dom}\left(\tau^{p}\right)$. Let $\varrho: F^{p} \rightarrow 2$ be such that $k \in A_{\beta} \Leftrightarrow \varrho(\beta)=1$. Let $n \leq n^{p}$ be maximal such that $k \in \bigcap_{\phi(\alpha)<n} A_{\alpha}$. By the property of $\phi$, the set $\left[\bigcap_{\phi(\alpha)<n} A_{\alpha} \backslash \bigcap_{\phi(\alpha) \leq n} A_{\alpha}\right] \cap L_{\varrho} \cap X$ is infinite, so we can choose a 
natural number $k^{\prime}$ in this set which is not in $\operatorname{ran}\left(\tau^{p}\right)$. Let $\tau^{q}=\tau^{p} \cup\left\{\left\langle k, k^{\prime}\right\rangle\right\}$, $F^{q}=F^{p}$, and $n^{q}=n^{p}$. Then $q \leq p$.

By a similar argument, for each $k \in X$, the set of all $p$ with $k \in \operatorname{ran}\left(\tau^{p}\right)$ is dense. It follows that $t: \omega \rightarrow X$ is a bijection.

We now prove that $t$ is an isomorphism between $\mathcal{F}$ and $\mathcal{F}\lceil X$ by showing that

(1) $t\left(A_{\beta}\right)={ }^{*} X \cap A_{\beta}$ for every $\beta \geq \delta$; and

(2) $t\left(\bigcap_{\phi(\alpha)<n} A_{\alpha}\right)={ }^{*} X \cap \bigcap_{\phi(\alpha)<n} A_{\alpha}$ for each $n<\omega$.

To see (1), fix $\beta \geq \delta$. There is $p \in G$ with $\beta \in F^{p}$. Let $k \notin \operatorname{dom}\left(\tau^{p}\right)$. There is $q \in G$ with $q \leq p$ and $k \in \tau^{q}$. Then $k \in A_{\beta} \Leftrightarrow \tau^{q}(k) \in A_{\beta} \Leftrightarrow t(k) \in A_{\beta}$. Similarly, if $k \in X \backslash \operatorname{ran}\left(\tau^{p}\right)$, then $k \in A_{\beta} \Leftrightarrow t^{-1}(k) \in A_{\beta}$. It follows that $t\left(A_{\beta} \backslash \operatorname{dom}\left(\tau^{p}\right)\right)=\left[X \cap A_{\beta}\right] \backslash \operatorname{ran}\left(\tau^{p}\right)$.

Now for (2), fix $n<\omega$. There is $p \in G$ with $n \leq n^{p}$. Let $k \notin \operatorname{dom}\left(\tau^{p}\right)$. There is $q \in G$ with $q \leq p$ and $k \in \tau^{q}$. Then $k \in \bigcap_{\phi(\alpha)<n} A_{\alpha} \Leftrightarrow \tau^{q}(k) \in \bigcap_{\phi(\alpha)<n} A_{a} \Leftrightarrow$ $t(k) \in \bigcap_{\phi(\alpha)<n} A_{a}$. The analogous statement is true for $k \in X \backslash \operatorname{ran}\left(\tau^{p}\right)$. It follows that $t\left(\bigcap_{\phi(\alpha)<n} A_{\alpha} \backslash \operatorname{dom}\left(\tau^{p}\right)\right)=\left[X \cap \bigcap_{\phi(\alpha)<n} A_{a}\right] \backslash \operatorname{ran}\left(\tau^{p}\right)$.

It follows that the bijections $t$ and $t^{-1}$ map elements of $\mathcal{F}$ to elements of its restriction to $X$, and vice versa. So $t$ witnesses that these two filters are isomorphic.

Now we define the poset $Q_{1}(\mathcal{A})$ forcing $\mathcal{F}$ to be isomorphic to the filter $\mathcal{F} \times \omega$ on $\omega^{2}$. Let $p=\left\langle\tau^{p}, F^{p}\right\rangle$ be in $Q_{1}(\mathcal{A})$ iff:

(a) $\tau^{p}$ is a finite one-to-one function from $\omega$ to $\omega^{2}$;

(b) $F^{p} \in\left[\omega_{1}\right]^{<\omega}$.

Define $q \leq p$ iff $\tau^{q} \supset \tau^{p}, F^{q} \supset F^{p}$, and $\forall n \in \operatorname{dom}\left(\tau^{q} \backslash \tau^{p}\right) \forall \beta \in F^{p}[n \in$ $\left.A_{\beta} \Leftrightarrow \tau^{q}(n) \in A_{\beta} \times \omega\right]$.

LEMma 4.4. The poset $Q_{1}(\mathcal{A})$ is $\sigma$-centered, and if $G$ is a $Q_{1}(\mathcal{A})$-generic filter, then $t=\bigcup\left\{\tau^{p}: p \in G\right\}$ is a bijection from $\omega$ to $\omega^{2}$ such that $t\left(A_{\beta}\right)={ }^{*}$ $A_{\beta} \times \omega$ for all $\beta \in \omega_{1}$. In particular, $t$ witnesses that in $V[G], \mathcal{F}$ is isomorphic to $\mathcal{F} \times \omega$.

Proof. Note that the collection $\left\{A_{\alpha} \times \omega: \alpha<\omega_{1}\right\}$, which generates the filter on $\omega^{2}$, is an independent family. Thus Lemma 4.4 follows by a proof similar to (and somewhat shorter than, since the complication of the finite-to-one function is not involved here) that of Lemma 4.3.

Now we describe the iteration, which is a finite support iteration $P_{\omega_{1}}$ of $\left\langle\dot{Q}_{\alpha}\right\rangle_{\alpha<\omega_{1}}$. Let the ground model $V$ satisfy CH. Let $Q_{0}=\operatorname{Fn}\left(\omega_{1} \times \omega, 2\right)$; i.e., $Q_{0}$ is the poset for adding $\omega_{1}$-many Cohen reals. If $G_{0}$ is $Q_{0}$-generic and $\alpha<\omega_{1}$, let $A_{\alpha}=\left\{n \in \omega: \bigcup G_{0}(\alpha, n)=1\right\}$. Then $\mathcal{A}=\left\{A_{\alpha}: \alpha<\omega_{1}\right\}$ is an independent family in $V\left[G_{0}\right]$. Let $\dot{Q}_{1}$ be a $Q_{0}$-name for the forcing $Q_{1}(\mathcal{A})$ of Lemma 4.4. Each $\dot{Q}_{\alpha}$ for $\alpha>1$ will be a name for a forcing $Q\left(\mathcal{A}, X_{\alpha}, \delta_{\alpha}, \phi_{\alpha}\right)$ 
as in Lemma 4.3 forcing $\mathcal{F}$ and its restriction to $X_{\alpha}$ to be isomorphic. Since $V \vDash \mathrm{CH}$, and each poset in the iteration is CCC and has size $\omega_{1}$, it follows that the final model satisfies $\mathrm{CH}$, and we can arrange the $X_{\alpha}$ 's to include (names for) every $X \in \mathcal{F}^{+}$in the final model. Thus in the end $\mathcal{F}$ will be homogeneous, and, thanks to $\dot{Q}_{1}$, will be isomorphic to $\mathcal{F} \times \omega$. It turns out nothing more need be done to obtain the additional property (iii) of Theorem 4.1.

However, a problem with the above simple outline is that, given that $X_{\alpha} \in \mathcal{F}^{+}$in $V^{P_{\alpha}}$, in order to continue the iteration we must show that there is forced to be some $\delta_{\alpha}<\omega_{1}$ and finite-to-one function $\phi_{\alpha}: \delta_{\alpha} \rightarrow \omega$ such that $Q\left(\mathcal{A}, X_{\alpha}, \delta_{\alpha}, \phi_{\alpha}\right)$ exists, i.e., the conclusion of Lemma 4.2 is satisfied. So we must show that $P_{\alpha}$ forces the hypothesis of Lemma 4.2.

To establish notation, let us describe the iteration more precisely. We will often abuse notation by letting sets be names for themselves, when this should cause no confusion, but we will also use $\dot{X}$ to denote a name for $X$ when we want to emphasize that we are talking about names.

First note that, without loss of generality, we can think of members of the iteration as having the form

$$
p=\left\langle\sigma^{p},\left\langle\tau_{1}^{p}, F_{1}^{p}\right\rangle,\left\langle\tau_{\gamma}^{p}, F_{\gamma}^{p}, n_{\gamma}^{p}\right\rangle_{\gamma \in D^{p}}\right\rangle
$$

where

(i) $\sigma^{p} \in \operatorname{Fn}\left(\omega_{1} \times \omega, 2\right)$;

(ii) $\tau_{1}^{p}$ is a finite one-to-one function from $\omega$ to $\omega^{2}$ and $F_{1}^{p} \in\left[\omega_{1}\right]^{<\omega}$;

(iii) $D^{p} \in\left[\omega_{1} \backslash 2\right]^{<\omega}$;

(iv) for each $\gamma \in D^{p}, \tau_{\gamma}^{p}$ is a finite one-to-one function from $\omega$ to $\omega$, $F_{\gamma}^{p} \in\left[\omega_{1}\right]^{<\omega}$, and $n_{\gamma}^{p}<\omega$. that:

Further, there is a sequence $\left(\dot{X}_{\alpha}, \dot{\delta}_{\alpha}, \dot{\phi}_{\alpha}\right), 1<\alpha<\omega_{1}$, of names such

(v) $\Vdash_{\alpha}$ " $\dot{X}_{\alpha} \in \mathcal{F}^{+}, \dot{\delta}_{\alpha}<\omega_{1}, \dot{\phi}_{\alpha}: \dot{\delta}_{\alpha} \rightarrow \omega$ is finite-to-one, and $\dot{X}_{\alpha}, \dot{\delta}_{\alpha}, \dot{\phi}_{\alpha}$ satisfy the conclusion of Lemma 4.2", and for each $\gamma \in D^{p}$,

(vi) $p\left\lceil\gamma \Vdash " p(\gamma) \in Q\left(\dot{\mathcal{A}}, \dot{X}_{\alpha}, \dot{\delta}, \dot{\phi}\right) "\right.$.

In particular this implies

(vii) $p\left\lceil\gamma \Vdash " \operatorname{ran}\left(\tau_{\gamma}^{p}\right) \subset \dot{X}_{\alpha}\right.$ and $F_{\gamma}^{p} \subset \omega_{1} \backslash \dot{\delta}_{\alpha} "$.

As indicated above, we can also assume that

(viii) $\Vdash_{\omega_{1}}$ " $X \in \mathcal{F}^{+} \Rightarrow \exists \alpha<\omega_{1}\left(X=\dot{X}_{\alpha}\right)$ ".

Let us also make the following simple but useful observation. If we change $p$ by extending $\sigma^{p}$ and doing nothing else, we get a stronger condition; it follows that $p \Vdash n \in A_{\beta} \Leftrightarrow \sigma^{p} \Vdash n \in A_{\beta} \Leftrightarrow\langle(\beta, n), 1\rangle \in \sigma^{p}$. 
In order to show that an iteration as described above actually exists, we need to show that in $V^{P_{\alpha}}$, any $X \in \mathcal{F}^{+}$satisfies the hypothesis of Lemma 4.2. Then if at stage $\alpha$ we are given some $X_{\alpha} \in \mathcal{F}^{+}$, we can conclude that there is a $\delta_{\alpha}<\omega_{1}$ and finite-to-one $\phi_{\alpha}: \delta_{\alpha} \rightarrow \omega$ such that $X_{\alpha}, \delta_{\alpha}$, and $\phi_{\alpha}$ satisfy the conclusion of Lemma 4.2 , and thus continue the iteration as described.

First we show, roughly speaking, that in $V^{P_{\alpha}}$, members of $\mathcal{F}^{+}$are not contained in an "orbit" of finitely many of the previously added isomorphisms $t_{\beta}: \omega \rightarrow X_{\beta}, 1<\beta<\alpha$, and $t_{1}: \omega \rightarrow \omega^{2}$. This will be needed later to show that we are free enough to extend conditions to force things we want to force.

Let $T$ be a collection of one-to-one functions from $\omega$ to $\omega$ or to $\omega^{2}$, and let $k \in \omega$. We say that $O(T, k) \subset \omega$ is the orbit of $k$ under $T$ if:

(i) whenever $t \in T, t: \omega \rightarrow \omega$, and $n \in O(T, k)$, then $t(n)$ and $t^{-1}(n)$ (if defined) are in $O(T, k)$;

(ii) whenever $t \in T, t: \omega \rightarrow \omega^{2}$, and $n \in O(T, k)$, then $\pi_{1}(t(n)) \in$ $O(T, k)$ and $t^{-1}(n, j) \in O(T, k)$ for all $j$;

(iii) $O(T, k)$ is the smallest set containing $k$ and satisfying (i) and (ii).

Note that $n \in O(T, k)$ iff there is a finite sequence $n_{0}, n_{1}, \ldots, n_{l}$ of natural numbers such that $n_{0}=k$ and $n_{l}=n$, and a finite sequence $t_{0}, t_{1}, \ldots, t_{l}$ of members of $T$ such that, for each $i<l$, either $n_{i+1}=t_{i}\left(n_{i}\right), n_{i}=t_{i+1}\left(n_{i+1}\right)$, $n_{i+1}=\pi_{1}\left(t_{i}\left(n_{i}\right)\right)$, or $n_{i}=\pi_{1}\left(t_{i+1}\left(n_{i+1}\right)\right)$.

Lemma 4.5. Let $t_{1}: \omega \rightarrow \omega^{2}$ be the bijection added by the first coordinate (i.e., by $\dot{Q}_{1}$ ) of the forcing $P_{\alpha}$, and for $1<\beta$ let $t_{\beta}: \omega \rightarrow \omega$ be that added by the $\beta$ th coordinate. Let $T$ be a finite subset of $\left\{t_{\beta}: 0<\beta<\alpha\right\}$. Then for each $k \in \omega$,

$$
\Vdash_{\alpha} " O(T, k) \notin \mathcal{F}^{+} " .
$$

Proof. Suppose not. Then for some $p \in P_{\alpha}, p \Vdash O(T, k) \in \mathcal{F}^{+}$. We assume $p$ has the form described above, and we may also assume $p \Vdash T=$ $\left\{t_{\gamma}: \gamma \in E\right\}$, where $E \in[\alpha]^{<\omega}$. Choose $\mu<\omega_{1}$ such that no $(\mu, n)$ is in $\operatorname{dom}\left(\sigma^{p}\right)$ and $p\left\lceil\gamma \Vdash \dot{\delta}_{\gamma} \leq \mu\right.$ for each $\gamma \in D^{p}$ (such a $\mu$ exists by CCC). Let $k^{\prime}<\omega$ be greater than $k$ and any integer mentioned in $\sigma^{p}$ or the $\tau_{\gamma}^{p}$ 's, $\gamma \in\{1\} \cup D^{p}$. Let $p^{\prime}$ be obtained from $p$ by adding $\langle(\mu, i), 0\rangle$ to $\sigma^{p}$ for each $i<k^{\prime}$, and adding $\mu$ to each $F_{\gamma}^{p}, \gamma \in\{1\} \cup D^{p}$. It is easy to check that $p^{\prime}$ is a condition stronger than $p$.

Since $p^{\prime} \Vdash O(T, k) \in \mathcal{F}^{+}$, there are $q \leq p^{\prime}$ and $m \geq k^{\prime}$ such that $q \Vdash m \in O(T, k) \cap A_{\mu}$. By extending $q$ if necessary, we may assume that there is a sequence $\left\langle n_{0}, n_{1}, \ldots, n_{l}\right\rangle$ of integers and a sequence $\left\langle\gamma_{0}, \gamma_{1}, \ldots, \gamma_{l}\right\rangle \in$ $E^{<\omega}$ such that this sequence of integers and the sequence of members of $T$ corresponding to the $\gamma_{i}$ 's witness that $m \in O(T, k)$ (i.e., $n_{0}=k, n_{l}=m$, 
etc...; see the discussion of $O(T, k)$ prior to the statement of this lemma). We may also assume that $q$, hence $\sigma^{q}$, decides whether or not $n_{i} \in A_{\mu}$, for all $i \leq l$, and if, e.g., $q \Vdash t_{\gamma_{i}}\left(n_{i}\right)=n_{i+1}$, then $\tau_{\gamma_{i}}^{q}\left(n_{i}\right)=n_{i+1}$. The following claim contradicts $q \Vdash m \in A_{\mu}$, proving the lemma.

Claim. For each $i \leq l, q \Vdash n_{i} \notin A_{\mu}$.

Proof of Claim. Since $\sigma^{p^{\prime}}(\langle\mu, k\rangle)=0, n_{0}=k$, and $q \leq p^{\prime}$, we have $q \Vdash n_{0} \notin A_{\mu}$. Suppose the claim is false, and let $i<l$ be least such that $q \Vdash n_{i+1} \in A_{\mu}$. Suppose, e.g., that, $\gamma_{i}=1$ and $\pi_{1}\left(\tau_{1}^{q}\left(n_{i}\right)\right)=n_{i+1}$ (all other cases, which we omit, are similar). Note that $q \Vdash n_{i+1} \in A_{\mu}$ and $q \leq p^{\prime}$ implies that $n_{i+1}$ is not mentioned by $\tau_{1}^{p}$, and so not by $\tau_{1}^{p^{\prime}}$ either. Thus $n_{i} \notin \operatorname{dom}\left(\tau_{1}^{p^{\prime}}\right)$. But now $q \leq p^{\prime}$ and $\mu \in F_{1}^{p^{\prime}}$ imply $q\left\lceil 1 \Vdash n_{i} \in A_{\mu} \Leftrightarrow \tau_{1}^{q}\left(n_{i}\right) \in\right.$ $A_{\mu} \times \omega \Leftrightarrow n_{i+1} \in A_{\mu}$. But by our choice of $i, q \Vdash$ " $n_{i} \notin A_{\mu}$ and $n_{i+1} \in A_{\mu}$ ", contradiction.

The following lemma shows that, under certain circumstances and for certain values of $\beta$ and $m$, we are free to extend two compatible conditions so that $m$ is forced to be in $A_{\beta}$, or not, as we wish.

Lemma 4.6. Let $\alpha<\omega_{1}$, let $q \in P_{\alpha}$, and let $M$ be a countable elementary submodel containing $q$ and $P_{\alpha}$. Let $p \in P_{\alpha}$ be compatible with $q$ (we do not require $p \in M$ however). Let $l$ be greater than any integer mentioned by $p$ (i.e., greater than any $n_{\mu}^{p}, \mu \in D^{p}$, or anything in the domain or range of $\sigma^{p}$ or of any $\left.\tau_{\mu}^{p}\right)$. Let $T=\left\{t_{\mu}: \mu \in D^{p} \cup\{1\}\right\}$, where $t_{\mu}$ is the isomorphism added by the $\mu$ th coordinate of the forcing. Suppose the following hold:

(i) $D^{q} \supset D^{p}$;

(ii) for each $\mu \in D^{p} \cup\{1\}$, $\tau_{\mu}^{q} \supset \tau_{\mu}^{p}$ and $F_{\mu}^{q} \supset F_{\mu}^{p} \cap M$;

(iii) for each $\mu \in D^{p}, n_{\mu}^{q} \geq n_{\mu}^{p}$;

(iv) for each $\mu \in D^{p}, q\left\lceil\mu\right.$ decides $\phi_{\mu}^{-1}(i)$ for each $i<n_{\mu}^{p}$ (i.e., there are finite sets $K_{\mu i}^{q}$ of ordinals, $i<n_{\mu}^{p}$, such that $q\left\lceil\mu \Vdash \phi_{\mu}^{-1}(i)=K_{\mu i}^{q}\right)$;

(v) $q \Vdash m \in \omega \backslash \bigcup_{k<l} O(T, k)$.

Then whenever $\varrho \in \operatorname{Fn}\left(\omega_{1} \backslash M, 2\right)$, there is a condition $s \leq p, q$ such that $\sigma^{s}(\langle\beta, m\rangle)=\varrho(\beta)$ for all $\beta \in \operatorname{dom}(\varrho)$.

Proof. Since $q$ and $p$ are compatible (by hypothesis), we can choose $r \leq q, p$ such that $\sigma^{r}$ decides whether or not $n \in A_{\beta}$ whenever:

(1) $n$ appears in the domain or range of some $\tau_{\mu}^{q}$ for $\mu \in D^{p} \cup\{1\}$, and

(2) $\beta \in F_{1}^{q} \cup \bigcup\left\{F_{\mu}^{q} \cup K_{\mu i}^{q}: \mu \in D^{p}, i<n_{\mu}^{p}\right\}$.

Let $O=O\left(\left\{\tau_{\mu}^{r}\right\}_{\mu \in D^{p} \cup\{1\}}, m\right)$. Note that $O$ contains $m$ and is finite (it would have to be contained in the union of $\{m\}$ and the finite set of integers mentioned in the domain and range of the $\tau_{\mu}^{r}$ s). Also $O \cap l=\emptyset$ since $r \Vdash m \notin \bigcup_{k<l} O(T, k)$. 
Fix $\varrho \in \operatorname{Fn}\left(\omega_{1} \backslash M, 2\right)$. We need to show that there is a condition $s \leq$ $p, q$ such that $\sigma^{s}(\langle\beta, m\rangle)=\varrho(\beta)$ for all $\beta \in \operatorname{dom}(\varrho)$. To this end, first let $\sigma \in \operatorname{Fn}\left(\omega_{1} \times \omega, 2\right)$ be such that:

(3) $\operatorname{dom}(\sigma)=\operatorname{dom}\left(\sigma^{r}\right) \cup(\operatorname{dom}(\varrho) \times O)$;

(4) $\sigma(\langle\beta, n\rangle)=\varrho(\beta)$ if $\beta \in \operatorname{dom}(\varrho)$ and $n \in O$;

(5) $\sigma(\langle\beta, n\rangle)=\sigma^{r}(\langle\beta, n\rangle)$ otherwise.

Now let

$$
s=\left\langle\sigma,\left\langle\tau_{1}^{q}, F_{1}^{q} \cup F_{1}^{p}\right\rangle,\left\langle\tau_{\mu}^{q}, F_{\mu}^{q} \cup F_{\mu}^{p}, n_{\mu}^{q}\right\rangle_{\mu \in D^{q}}\right\rangle .
$$

By (4) above, $s$ (if it is a condition) forces what we want. It remains to prove that $s$ is a condition extending both $q$ and $p$.

Note that $\sigma \supset \sigma^{q} \cup \sigma^{p}$, since:

(6) $\sigma^{r} \supset \sigma^{q} \cup \sigma^{p}$;

(7) $\sigma=\sigma^{r}$ outside of $\operatorname{dom}(\varrho) \times O$;

(8) $\operatorname{dom}(\varrho) \cap \operatorname{dom}\left(\sigma^{q}\right)=\emptyset$ (recall $q \in M$ and $\left.\operatorname{dom}(\varrho) \subset \omega_{1} \backslash M\right)$; and

(9) $O \cap l=\emptyset$.

So $s$ can be thought of as $q$ with $\sigma^{q}$ extended, the $F_{\mu}^{q}$ 's enlarged, but the $\tau_{\mu}^{q}$ 's left the same. Also recall $F_{\mu}^{q} \supset F_{\mu}^{p} \cap M$ for every $\mu \in D^{p} \cup\{1\}$, so $F_{\mu}^{s} \backslash F_{\mu}^{q} \subset \omega_{1} \backslash M$ (this is needed to show $s\left\lceil\mu \Vdash F_{\mu}^{s} \subset \omega_{1} \backslash \dot{\delta}_{\mu}\right.$ ). It easily follows that $s$ is a condition and $s \leq q$.

It remains to prove that $s \leq p$. From (i)-(iii) and the definition of $s$, we have the necessary containments, and $\tau_{\mu}^{s}=\tau_{\mu}^{q}$, so what we need to show is that $s\lceil\mu$ forces:

(a) $\forall n \in \operatorname{dom}\left(\tau_{\mu}^{q} \backslash \tau_{\mu}^{p}\right) \forall \beta \in F_{\mu}^{p}\left[n \in A_{\beta} \Leftrightarrow \tau_{\mu}^{q}(n) \in A_{\beta}\right]$;

(b) $\forall n \in \operatorname{dom}\left(\tau_{\mu}^{q} \backslash \tau_{\mu}^{p}\right) \forall k \leq n_{\mu}^{p}\left[n \in \bigcap_{\phi_{\mu}(\beta)<k} A_{\beta} \Leftrightarrow \tau^{q}(n) \in \bigcap_{\phi_{\mu}(\beta)<k} A_{\beta}\right]$ for each $\mu \in D^{p}$, and

(c) $\forall n \in \operatorname{dom}\left(\tau_{1}^{q} \backslash \tau_{1}^{p}\right) \forall \beta \in F_{1}^{p}\left[n \in A_{\beta} \Leftrightarrow \tau_{1}^{q}(n) \in A_{\beta} \times \omega\right]$.

Proof of $(a)$. Let $n \in \operatorname{dom}\left(\tau_{\mu}^{q} \backslash \tau_{\mu}^{p}\right), \beta \in F_{\mu}^{p}$, where $\mu \in D^{p}$. Since $\tau_{\mu}^{q}(n)=\tau_{\mu}^{r}(n)$, by the definition of $O$ we have $n \in O \Leftrightarrow \tau_{\mu}^{q}(n) \in O$. So if $\langle\beta, n\rangle$ is in $\operatorname{dom}(\varrho) \times O$, then so is $\left\langle\beta, \tau_{\mu}^{q}(n)\right\rangle$ and by (4) above, $\sigma(=s\lceil 1)$ forces " $n \in A_{\beta} \Leftrightarrow \varrho(\beta)=1 \Leftrightarrow \tau_{\mu}^{q}(n) \in A_{\beta}$ ". On the other hand, if $\langle\beta, n\rangle$ is not in $\operatorname{dom}(\varrho) \times O$, neither is $\left\langle\beta, \tau_{\mu}^{q}(n)\right\rangle$, and so $\sigma$ and $\sigma^{r}$ agree on these two values. Since $r \leq p$ and $n \in \operatorname{dom}\left(\tau_{\mu}^{r} \backslash \tau_{\mu}^{p}\right)$, we must have $\sigma^{r} \Vdash$ " $n \in A_{\beta} \Leftrightarrow$ $\tau_{\mu}^{q}(n) \in A_{\beta}$ ". So $\sigma$, hence $s\lceil\mu$, forces this too.

Proof of $(c)$. This proof is virtually the same as for (a), if we put $\mu=1$ and put $\pi_{1}\left(\tau_{1}^{q}(n)\right)$ in place of $\tau_{\mu}^{q}(n)$. 
Proof of (b). Let $n \in \operatorname{dom}\left(\tau_{\mu}^{q} \backslash \tau_{\mu}^{p}\right)$ and $k \leq n_{\mu}$. By (iv), and since $r, s \leq q$,

$$
r\left\lceil\mu, s\left\lceil\mu \Vdash \text { " } \phi_{\mu}(\beta)<k \Leftrightarrow \beta \in \bigcup_{i<k} K_{\mu i}^{q} " .\right.\right.
$$

Since $q \in M$ and $\operatorname{dom}(\varrho) \cap M=\emptyset$, we have $\operatorname{dom}(\varrho) \cap \bigcup_{i<k} K_{\mu i}^{q}=\emptyset$. By the definition of $r, \sigma^{r}$ decides the values of $\langle\beta, n\rangle$ and $\left\langle\beta, \tau_{\mu}^{q}(n)\right\rangle$ for any $\beta \in \bigcup_{i<k} K_{\mu i}^{q}$, and by definition $\sigma(=s\lceil 1)$ decides these values the same way. It follows that $r\left\lceil\mu \Vdash " n \in \bigcap_{\phi_{\mu}(\beta)<k} A_{\beta} " \Leftrightarrow s\left\lceil\mu \Vdash " n \in \bigcap_{\phi_{\mu}(\beta)<k} A_{\beta}\right.\right.$ " and $r\left\lceil\mu \Vdash\right.$ " $\tau_{\mu}^{q}(n) \in \bigcap_{\phi_{\mu}(\beta)<k} A_{\beta} " \Leftrightarrow s\left\lceil\mu \Vdash " \tau_{\mu}^{q}(n) \in \bigcap_{\phi_{\mu}(\beta)<k} A_{\beta}\right.$ ". Now since $r\lceil\mu$ forces

$$
" n \in \bigcap_{\phi_{\mu}(\beta)<k} A_{\beta} \Leftrightarrow \tau_{\mu}^{q}(n) \in \bigcap_{\phi_{\mu}(\beta)<k} A_{\beta} "
$$

(since $n \in \operatorname{dom}\left(\tau_{\mu}^{r} \backslash \tau_{\mu}^{p}\right)$ and $r \leq p$ ) so does $s\lceil\mu$, and we are done.

The next lemma shows that the hypothesis of Lemma 4.2 is satisfied with respect to the independent family $\mathcal{A}$ added by the first factor.

Lemma 4.7. Let $\alpha<\omega_{1}$. Suppose $\Vdash_{\alpha} \dot{X} \in \mathcal{F}^{+}$. Then there exists $\gamma<$ $\omega_{1}$ such that $\Vdash_{\alpha} \dot{X} \cap L_{\varrho} \neq \emptyset$ for every $\varrho \in \operatorname{Fn}\left(\omega_{1} \backslash \gamma, 2\right)$ (where $L_{\varrho}=$ $\left.\bigcap_{\beta \in \operatorname{dom}(\varrho)} A_{\beta}^{\varrho(\beta)}\right)$.

Proof. If not, then for each $\gamma<\omega_{1}$, there are $p_{\gamma} \in P_{\alpha}$ and $\varrho_{\gamma} \in$ $\operatorname{Fn}\left(\omega_{1} \backslash \gamma, 2\right)$ such that $p_{\gamma} \Vdash \dot{X} \cap L_{\varrho_{\gamma}}=\emptyset$. Say

$$
p_{\gamma}=\left\langle\sigma^{\gamma},\left\langle\tau_{1}^{\gamma}, F_{1}^{\gamma}\right\rangle,\left\langle\tau_{\mu}^{\gamma}, F_{\mu}^{\gamma}, n_{\mu}^{\gamma}\right\rangle_{\mu \in D^{\gamma}}\right\rangle \text {. }
$$

We may assume:

(i) the $\sigma^{\gamma}$ 's form a $\Delta$-system with root $\sigma$;

(ii) $\exists D \subset \alpha$ such that $D^{\gamma}=D$ for all $\gamma$;

(iii) for all $\mu \in D \cup\{1\}$, there is $\tau_{\mu}$ such that $\tau_{\mu}^{\gamma}=\tau_{\mu}$ for all $\gamma$, and for all $\mu \in D$, there is $n_{\mu}$ such that $n_{\mu}^{\gamma}=n_{\mu}$ for all $\gamma$;

(iv) for all $\mu \in D \cup\{1\}$, the $F_{\mu}^{\gamma}$ 's form a $\Delta$-system with root $F_{\mu}$.

Let $p=\left\langle\sigma,\left\langle\tau_{1}, F_{1}\right\rangle,\left\langle\tau_{\mu}, F_{\mu}, n_{\mu}\right\rangle_{\mu \in D}\right\rangle$. Let us write $q \supset p$ if containment holds on each coordinate, i.e., $\sigma^{q} \supset \sigma, \tau_{1}^{q} \supset \tau_{1}$, etc. Note that this does not necessarily imply that $q \leq p$ in the sense of the poset $P_{\alpha}$.

Let $M$ be a countable elementary submodel containing $p, \dot{X}, P_{\alpha}$, etc. Choose $\gamma \in \omega_{1} \backslash M$ such that, for each $\mu \in D \cup\{1\}$,

$$
\left[\left(F_{\mu}^{\gamma} \backslash F_{\mu}\right) \cup \operatorname{dom}\left(\sigma^{\gamma} \backslash \sigma\right)\right] \cap M=\emptyset .
$$

Let $l$ be greater than any integer mentioned by $p_{\gamma}$ (i.e., greater than any $n_{\mu}, \mu \in D \cup\{1\}$, or anything in the domain or range of $\sigma^{\gamma}$ or of any $\left.\tau_{\mu}^{\gamma}\right)$.

Let $T=\left\{t_{\mu}: \mu \in D \cup\{1\}\right\}$, where $t_{\mu}$ is the isomorphism added by the $\mu$ th coordinate of the forcing. Since $\Vdash_{\alpha} \dot{X} \in \mathcal{F}^{+}$, by Lemma 4.5 there are 
$r \leq p_{\gamma}$ and $m \in \omega$ such that

$$
r \Vdash \text { " } m \in \dot{X} \backslash \bigcup_{k<l} O(T, k) " .
$$

Consider a maximal antichain of conditions $q \supset p$ such that:

(a) $q \Vdash$ " $m \in \dot{X} \backslash \bigcup_{k<l} O(T, k)$ ";

(b) for each $\mu \in D \cup\{1\}, q\left\lceil\mu\right.$ decides $\phi_{\mu}^{-1}(i)$ for each $i<n_{\mu}$ (i.e., there are finite sets $K_{\mu i}^{q}$ of ordinals, $i<n_{\mu}$, such that $q\left\lceil\mu \Vdash \phi_{\mu}^{-1}(i)=K_{\mu i}^{q}\right)$.

Any such maximal antichain must contain a condition compatible with $r$. By CCC, and elementarity of $M$, there is such a maximal antichain in $M$, and hence we can choose a $q \supset p$ in $M$ satisfying (a) and (b) which is compatible with $r$.

Note that the conditions of Lemma 4.6 are satisfied for this $q$ with $p=p_{\gamma}$ and $\varrho=\varrho_{\gamma}$. So there is $s \leq p, q$ with $\sigma^{s}(\langle\beta, m\rangle)=\varrho_{\gamma}(\beta)$ for all $\beta \in \operatorname{dom}\left(\varrho_{\gamma}\right)$. Then $s \Vdash m \in \dot{X} \cap L_{\varrho_{\gamma}}$. This contradicts $p_{\gamma} \Vdash \dot{X} \cap L_{\varrho_{\gamma}}=\emptyset$, which completes the proof.

At this point, we can see that the iteration can be continued as claimed, with $\dot{Q}_{\alpha}, \alpha>1$, forcing $\mathcal{F}$ to be isomorphic to its restriction to $X_{\alpha}$. Since each $X \in \mathcal{F}^{+}$in the final model is named by some $\dot{X}_{\alpha}, \alpha<\omega_{1}, \mathcal{F}$ is homogeneous in $V^{P_{\omega_{1}}}$. The forcing $\dot{Q}_{1}$ assures us that $\mathcal{F}$ is isomorphic to $\mathcal{F} \times \omega$.

It remains to prove that $\mathcal{F}$ also satisfies condition (iii) in Theorem 4.1. Let us note that any subset $I$ of $\omega$ which appears by stage $1<\alpha<\omega_{1}$ and is in $\mathcal{F}^{+}$in $V^{P_{\alpha}}$ remains in $\mathcal{F}^{+}$in $V^{P_{\omega_{1}}}$ (since for each finite $H \subset \omega_{1}$, the statement " $I \cap \bigcap_{\beta \in H} A_{\beta} \neq \emptyset$ " is absolute for transitive models of set theory).

Therefore, since any $f: \omega \rightarrow \omega$ in the final model appears in some initial stage, it suffices to prove that condition (iii) holds at every stage $1<\alpha<\omega_{1}$. So suppose

$$
\Vdash_{\alpha} " \dot{f}: \omega \rightarrow \omega \text { and } \forall F \in \mathcal{F}(\dot{f}(F) \text { is unbounded }) " .
$$

Let $M$ be a countable elementary submodel containing $P_{\alpha}, \dot{f}, \ldots$ Let $M \cap \omega_{1}=\left\{\alpha_{0}, \alpha_{1}, \ldots\right\}$

Let $T_{k}=\left\{t_{\alpha_{l}}: l<k, 1 \leq \alpha_{l}<\alpha\right\}$. Let $a_{\emptyset}=\emptyset$. By induction on $k \geq 1$, we can define, for each $\sigma \in \omega^{k}$, conditions $a_{\sigma} \in M \cap P_{\alpha}$, and $i_{\sigma}, j_{\sigma} \in \omega$ such that:

(a) $a_{\sigma} \Vdash " \dot{f}\left(i_{\sigma}\right)=j_{\sigma}$ and $i_{\sigma} \in \bigcap_{l<|\sigma|} A_{\alpha_{l}} \backslash \bigcup_{m<|\sigma|} O\left(T_{|\sigma|}, m\right) "$;

(b) $i_{\sigma \frown\langle n\rangle}>i_{\sigma}$ and $j_{\sigma \frown\langle n\rangle}>j_{\sigma}$;

(c) $D^{a_{\sigma}} \supset\left\{\alpha_{l}: l<|\sigma|, 1<\alpha_{l}<\alpha\right\}$;

(d) for each $\mu \in D^{a_{\sigma}}, n_{\mu}^{a_{\sigma}} \geq|\sigma|$, and there is $\delta_{\mu}^{a_{\sigma}}<\omega_{1}$ such that $a_{\sigma}\left\lceil\mu \Vdash \dot{\delta}_{\mu}=\delta_{\mu}^{\alpha_{\sigma}}\right.$; 
(e) for each $\mu \in D^{a_{\sigma}}$, for each $i<|\sigma|, a_{\sigma}\left\lceil\mu\right.$ decides the value of $\phi_{\mu}^{-1}(i)$;

(f) for each $\mu \in D^{a_{\sigma}} \cup\{1\}$, $\operatorname{dom}\left(\tau_{\mu}^{a_{\sigma}}\right) \supset|\sigma|$;

(g) $F_{1}^{a_{\sigma}} \supset\left\{\alpha_{l}: l<|\sigma|\right\}$ and for each $\mu \in D^{\alpha_{\sigma}}, F_{\mu}^{a_{\sigma}} \supset\left\{\alpha_{l}: l<|\sigma|\right.$, $\left.\delta_{\mu}^{a_{\sigma}} \leq \alpha_{l}<M \cap \omega_{1}\right\}$

(h) $a_{\sigma} \leq a_{\sigma \uparrow l}$ for each $l \leq|\sigma|$;

(j) $\left\{a_{\sigma} \frown\langle n\rangle: n<\omega\right\}$ is a maximal antichain below $a_{\sigma}$.

Note that (a) and (b) can be obtained because $\dot{f}$ is forced to be unbounded on every $F \in \mathcal{F}$. Then (c)-(g) can be obtained simply by extending as necessary. So given that $a_{\sigma}$ has been defined, there is a maximal antichain of conditions below $a_{\sigma}$ satisfying (a) $-(\mathrm{g})$, and by $\mathrm{CCC}$, there is one in $M$; hence $(\mathrm{h})$ and $(\mathrm{j})$ can be obtained as well.

Note that for any $P_{\alpha}$-generic $G$, there is a unique $h \in \omega^{\omega}$ with $a_{h\lceil n} \in G$ for each $n \in \omega$. So there a corresponding set $I=\left\{i_{h \uparrow n}: n<\omega\right\}$ in $V[G]$. By (a) and (b), $\dot{f}_{G}$ is strictly increasing on $I$.

Let $\dot{I}$ be a $P_{\alpha}$-name for $I$. It remains to prove that $\Vdash_{\alpha} \dot{I} \in \mathcal{F}^{+}$. Suppose not. Then there is some $p \in P_{\alpha}$ and finite subset $H$ of $\omega_{1}$ such that $p \Vdash$ "I $\cap \bigcap_{\beta \in H} A_{\beta}=\emptyset "$.

Note that there is some $a_{\sigma}$ compatible with $p$ for $\sigma$ of any prescribed length. So we can choose $a_{\sigma}$ compatible with $p$ so that:

(k) $|\sigma|$ is greater than any integer mentioned in $p$;

(l) $\left\{\alpha_{l}: l<|\sigma|\right\} \supset D^{p} \cup(H \cap M) \cup \bigcup\left\{F_{\mu}^{p} \cap M: \mu \in D^{p}\right\}$.

Then from (l) and (a) we have:

(m) $a_{\sigma} \Vdash i_{\sigma} \in \bigcap_{\gamma \in H \cap M} A_{\gamma}$.

By (c) and (l) we have:

(n) $D^{a_{\sigma}} \supset D^{p}$.

By (k), (n), (f), and compatibility we have:

(o) for each $\mu \in D^{p} \cup\{1\}, \tau_{\mu}^{a_{\sigma}} \supset \tau_{\mu}^{p}$.

By (d), (g), (l), and compatibility we have:

(p) for each $\mu \in D^{p} \cup\{1\}, F_{\mu}^{a_{\sigma}} \supset F_{\mu}^{p} \cap M$, and $n_{\mu}^{a_{\sigma}} \geq n_{\mu}^{p}$ for each $\mu \in D^{p}$.

It follows from (a), (e), (k), (n), (o), and (p) that the conditions of Lemma 4.6 are satisfied for this $p$ with $q=a^{\sigma}, m=i_{\sigma}$, and $l=|\sigma|$. So, there exists $s \leq a_{\sigma}, p$ with $\sigma^{s}\left(\left\langle\beta, i_{\sigma}\right\rangle\right)=1$ for each $\beta \in H \backslash M$. Then by $(\mathrm{m})$ and $s \leq a_{\sigma}$, we have

$$
s \Vdash i_{\sigma} \in \dot{I} \cap \bigcap_{\beta \in H} A_{\beta} .
$$

This contradicts $s \leq p$, and completes the proof of Theorem 4.1. 
We wish to remark here that there is a theorem of W. H. Woodin which suggests that the Continuum Hypothesis implies that a filter with the properties given in Theorem 4.1 exists. In particular he has shown that if $\phi$ is a $\Sigma_{1}^{2}$ sentence (i.e. a sentence of the form $\exists \mathcal{F} \subseteq P(\omega) Q_{1} x_{1} \ldots Q_{k} x_{k} \psi\left(\mathcal{F}, x_{1}, \ldots\right.$ $\left.\ldots, x_{k}\right)$ where $Q_{i}$ is a quantifier over the reals and $\psi$ is a quantifier free formula) then, provided that certain large cardinals exist (a supercompact is sufficient), if $\phi$ can be forced to be true, then it holds in any forcing extension satisfying the Continuum Hypothesis. In other words, if $\phi$ is not a consequence of the Continuum Hypothesis, then the proof cannot be purely a forcing argument. It is not difficult to show that the existence of a filter with the properties of Theorem 4.1 is a statement of this form. If the existence of such a filter were not a consequence of the Continuum Hypothesis then this would be of independent interest.

5. Concluding remarks. The topologies of our spaces $T_{\alpha}$ are determined by the filter $\mathcal{F}$ on $\omega$, and by the " $\cup_{\mathcal{F}}$ construction" for defining the neighborhoods of the point $\infty$ which we added to the countably many copies $\{n\} \times T_{\alpha}, n<\omega$, of $T_{\alpha}$, in order to construct $T_{\alpha+1}$ from $T_{\alpha}$. A different natural way of defining the topology of $T_{\alpha+1}$ is to declare $N$ to be a neighborhood of $\infty$ iff $\infty \in N$ and $N$ contains a neighborhood of the point $(n, \infty)$ of maximal rank in $\{n\} \times T_{\alpha}$, for $\mathcal{F}$-many $n \in \omega$. Let $T_{\alpha}^{\prime}$ be the spaces obtained by defining them inductively this way, i.e., just like the $T_{\alpha}$ 's but using this finer neighborhood base for $\infty$. Then $T_{2}^{\prime}$ is the same as $T_{2}$, i.e., it is homeomorphic to the space $\omega \cup\{\mathcal{F}\}$ with $\omega$ the set of isolated points and a neighborhood of $\mathcal{F}$ having the form $F \cup\{\mathcal{F}\}, F \in \mathcal{F}$. But $T_{3}^{\prime}$ is different; in particular, the restriction of the neighborhood filter of $\infty$ to the isolated points is isomorphic to the filter $\mathcal{F}^{2}$ on $\omega^{2}$ defined by

$$
\mathcal{F}^{2}=\left\{A \subset \omega^{2}:\{n:\{m:(n, m) \in A\} \in \mathcal{F}\} \in \mathcal{F}\right\} .
$$

A filter $\mathcal{F}$ is said to be idempotent if it is isomorphic to $\mathcal{F}^{2}$.

The following result shows that, while the $T_{\alpha}^{\prime}$ construction does yield a 3 -Toronto space in ZFC (iff $\mathcal{F}$ is an ultrafilter), $T_{\alpha}^{\prime}$ is never an $\alpha$-Toronto space if $\alpha \geq 4$.

Theorem 5.1. Let $\mathcal{F}$ be a filter on $\omega$, and let $T_{\alpha}^{\prime}$, $\alpha$ an ordinal, be the spaces defined as above. Then:

(a) $T_{3}^{\prime}$ is a 3 -Toronto space iff $\mathcal{F}$ is an ultrafilter;

(b) $T_{\alpha}^{\prime}$ is not an $\alpha$-Toronto space if $\alpha \geq 4$.

Proof. (a) We can consider $T_{3}^{\prime}$ as the set $(\omega \times(\omega+1)) \cup\{\infty\}$, where $\omega \times \omega$ is the set of isolated points, each $\langle n, \omega\rangle$ has a neighborhood base of sets of the form $\{n\} \times(F \cup\{\omega\}), F \in \mathcal{F}$, and a neighborhood of $\infty$ consists of $\infty$ together with neighborhoods of $\langle n, \omega\rangle$ for $\mathcal{F}$-many $n$. 
Suppose $\mathcal{F}$ is an ultrafilter and $X \subset T_{3}^{\prime}$ has rank 3 . Then for $\mathcal{F}$-many $n$, it must be the case that there are $F_{n} \in \mathcal{F}$ such that $\{n\} \times\left(F_{n} \cup\{\omega\}\right) \subset X$. Now, using the easily verified fact that any ultrafilter is homogeneous, it is not difficult to prove that $X \cong T_{3}^{\prime}$.

Suppose on the other hand that $\mathcal{F}$ is not an ultrafilter. Let $A \subset \omega$ be such that $A, \omega \backslash A \in \mathcal{F}^{+}$. Let $X=T_{3}^{\prime} \backslash(A \times\{\omega\})$. Then $X$ has rank 3. But the subset $A \times \omega$ of $X$ is a set of isolated points whose only limit point in $X$ is $\infty$, while in $T_{3}^{\prime}$ any set of isolated points clustering at $\infty$ must also have cluster points of the form $\langle n, \omega\rangle$. So $X$ is not homeomorphic to $T_{3}^{\prime}$.

(b) Let $\alpha \geq 4$, and consider removing the level one points from a neighborhood of some level 2 point of $T_{\alpha}^{\prime}$. By the discussion preceding the theorem, in the resulting subspace this level 2 point of $T_{\alpha}^{\prime}$ becomes a level one point with a neighborhood filter isomorphic to $\mathcal{F}^{2}$. Thus it must be the case that $\mathcal{F}$ is an idempotent filter. Frolík showed in $[F]$ that no ultrafilter is idempotent, so $\mathcal{F}$ is not an ultrafilter. Note that any set of isolated points of $T_{\alpha}^{\prime}$ which has a limit point at level 2 must also have a limit point at level 1. But, using an idea from the proof of part (a), we see that there is a subspace of $T_{\alpha}^{\prime}$ of rank $\alpha$ which fails to have this property. Hence $T_{\alpha}^{\prime}$ is not $\alpha$-Toronto.

Remark. An idempotent filter on $\omega$ has been constructed in ZFC by M. Katětov $[\mathrm{Ka}]$, and a homogeneous one (also in ZFC) by J. Steprāns (unpublished note). The latter result answers a question of Steprāns in [S].

\section{References}

[F] Z. Frolík, Fixed point maps of $\beta N$, Bull. Amer. Math. Soc. 74 (1968), 187-191.

[Ka] M. Katětov, On idempotent filters, Časopis Pěst. Mat. 102 (1977), 412-418.

[Ku] K. Kunen, Set Theory, North-Holland, Amsterdam, 1980.

[S] J. Steprāns, Steprāns' problems, in: Open Problems in Topology, J. van Mill and G. M. Reed (eds.), North-Holland, Amsterdam, 1990, 13-20.

Department of Mathematics

Auburn University

Auburn, AL 36849, U.S.A.

E-mail: garyg@auburn.edu
Department of Mathematics University of Toronto Toronto, Ontario M5S 1A1, Canada E-mail: justin@math.toronto.edu 